\title{
Maternal weight, gestational weight gain and preschool wheezing: the Generation R Study
}

\author{
Elisabeth T.M. Leermakers ${ }^{1,2,3,4}$, Agnes M.M. Sonnenschein-van der Voort ${ }^{1,2,5}$, \\ Romy Gaillard ${ }^{1,2,4}$, Albert Hofman², Johan C. de Jongste ${ }^{4}$, Vincent W.V. Jaddoe ${ }^{1,2,4}$ \\ and Liesbeth Duijts $1,2,5,6$
}

Affiliations: 'The Generation R Study Group, Erasmus Medical Center, Rotterdam, '2Dept of Epidemiology, Erasmus Medical Center, Rotterdam, ${ }^{3}$ ErasmusAGE, Dept of Epidemiology, Erasmus Medical Center, Rotterdam, ${ }^{4}$ Dept of Paediatrics, Erasmus Medical Center, Rotterdam, ${ }^{5}$ Dept of Paediatrics, Division of Respiratory Medicine, Erasmus Medical Center, Rotterdam, and ' $D e p t$ of Paediatrics, Division of Neonatology, Erasmus Medical Center, Rotterdam, The Netherlands.

Correspondence: L. Duijts, Erasmus Medical Center - Sophia Children's Hospital, Sp-3435, P0 Box 2060, 3000 CB Rotterdam, The Netherlands. E-mail: l.duijtsderasmusmc.nl

ABSTRACT We studied the associations of maternal pre-pregnancy body mass index and gestational weight gain with risks of preschool wheezing in offspring and explored the role of growth, infectious and atopic mechanisms.

This substudy of 4656 children was embedded in a population-based birth cohort. Information about maternal pre-pregnancy weight, gestational weight gain and wheezing at the ages of 1-4 years was obtained by either physical measurements or questionnaires.

Among mothers with a history of asthma or atopy, maternal pre-pregnancy obesity was associated with an overall increased risk of preschool wheezing (odds ratio 1.47, 95\% confidence interval 1.12-1.95). Also, each standard deviation increase of gestational weight gain was associated with an increased overall risk of preschool wheezing $(1.09,1.04-1.14)$, was independent of pre-pregnancy body mass index and was not different between mothers with and without a history of asthma or atopy. Child's growth, respiratory tract infections or eczema did not alter the results.

Mothers with pre-pregnancy obesity and a history of asthma or atopy, and mothers with higher gestational weight gain showed higher risks of wheezing in their offspring. These associations could not be explained by growth, infectious or atopic mechanisms. Further research is needed to identify underlying mechanisms and long-term consequences.

@ERSpublications

Pre-pregnancy obesity associated with increased risk of wheezing in offspring, if mothers have history of asthma or atopy http://ow.ly/nDG1u

This article has supplementary material available from www.erj.ersjournals.com

Received: Sept 182012 | Accepted after revision: Feb 142013 | First published online: March 072013

Support statement: The general design of the Generation R Study was made possible by financial support from the Erasmus Medical Center, Rotterdam, the Erasmus University Rotterdam and the Netherlands Organization for Health Research and Development. V. Jaddoe received an additional grant from the Netherlands Organization for Health Research and Development (ZonMw 90700303, 916.10159). L. Duijts is the recipient of a European Respiratory Society/ Marie Curie Joint Research Fellowship (number MC 1226-2009). The research leading to these results has received funding from the European Respiratory Society and the European Community's Seventh Framework Programme FP7/ 2007-2013 - Marie Curie Actions under grant agreement RESPIRE, PCOFUND-GA-2008-229571 and from the seventh framework programme, project CHICOS (HEALTH-F2-2009-241504).

Conflict of interest: Disclosures can be found alongside the online version of this article at www.erj.ersjournals.com

Copyright @ERS 2013 


\section{Introduction}

Maternal pre-pregnancy obesity is suggested to be associated with childhood asthma symptoms [1-6]. These studies mostly focused on maternal weight before or in early pregnancy and have inconsistent results regarding the role of maternal history of asthma or atopy. Mechanisms underlying the association between maternal pre-pregnancy obesity and childhood asthma symptoms are not known, but might include the child's growth, and infectious and atopic mechanisms. Maternal pre-pregnancy obesity seems to be associated with a difference in birthweight and gestational age at the time of delivery $[7,8]$ and might adversely affect pulmonary development of the fetus, leading to relatively smaller airways, impaired lung function, and asthma symptoms in childhood [9-11]. Childhood growth might modify the association of pre-pregnancy obesity with preschool wheezing [10, 12]. Another mechanism might be that proinflammatory cytokines levels are increased in obese mothers, which might affect the development of the fetal immune system and the risk of infectious and atopic diseases post-natally [13-16].

To date, the effect of gestational weight gain, which tends to be inversely associated with pre-pregnancy maternal body mass index (BMI), on the development of asthma symptoms has not been extensively studied. Gestational weight gain may modify the association of maternal BMI with wheezing, but could also be a risk in its own right. We hypothesise that maternal pre-pregnancy weight and gestational weight gain independently lead to increased risks of childhood wheezing. Studies focused on the associations of maternal pre-pregnancy obesity and gestational weight gain with childhood asthma symptoms, including potential underlying mechanisms, are important to identify specific adverse fetal exposures in critical periods in which airways and lungs develop. Therefore, first, we examined in a population-based prospective cohort study among 4656 children, the associations of maternal pre-pregnancy BMI and gestational weight gain with the risk of asthma symptoms including wheezing in preschool children. Secondly, we explored if any association could be explained by child's growth, infectious and atopic mechanisms, and if these associations were modified by family history of asthma or atopy.

\section{Methods}

Design

This study was embedded in the Generation R Study, a population-based prospective cohort study from early fetal life onwards in Rotterdam, the Netherlands [17]. The study has been approved by the Medical Ethical Committee of the Erasmus Medical Centre in Rotterdam. Written informed consent was obtained from all participants. A total of 4656 mothers and their children were included for the current analyses (fig. 1). The analyses with gestational weight gain as exposure were conducted in a slightly smaller sample of 4535 mothers and children.

\section{Maternal anthropometrics, obesity and weight gain during pregnancy}

Maternal anthropometrics (height and weight) were measured in the first, second and third trimester of pregnancy at one of the research centres [18]. Pre-pregnancy BMI $\left(\mathrm{kg} \cdot \mathrm{m}^{-2}\right)$ was calculated using prepregnancy weight as recorded by the mother and height $(\mathrm{cm})$ measured at enrolment and was categorised into four categories (underweight, $<20 \mathrm{~kg} \cdot \mathrm{m}^{-2}$; normal weight, $20-24.9 \mathrm{~kg} \cdot \mathrm{m}^{-2}$; overweight, $25-29.9 \mathrm{~kg} \cdot \mathrm{m}^{-2}$; obese, $\left.\geqslant 30 \mathrm{~kg} \cdot \mathrm{m}^{-2}\right)$. As enrolment in our study was in pregnancy, we were not able to measure maternal weight before pregnancy. Therefore, we obtained information about maternal weight just before pregnancy by the first questionnaire. In our population for analysis, 52\% of all females were enrolled before a gestational age of 14 weeks. Correlation of pre-pregnancy weight obtained by questionnaire, and weight measured in first trimester of pregnancy was very good (Pearson correlation $0.95 ; \mathrm{p}<0.001$ ). The agreement between prepregnancy BMI categories and BMI categories at intake was good (Cohen's kappa 0.62; $\mathrm{p}<0.0001$ ) and the Bland-Altman plot (fig. S1) showed no evidence of systematic bias.

We defined gestational weight gain as the difference between weight before pregnancy and weight in third trimester (measured without heavy clothing at a median of 30.2 weeks of gestational age (2.5-97.5\% range, 28.5-32.8 weeks)). This information was available for 4535 mothers. Standard deviation scores for gestational weight gain were created and used in the model as a continuous variable $[8,19]$.

\section{Respiratory symptoms}

Information on wheezing (no or yes) was obtained by questionnaires, adapted from the International Study on Asthma and Allergy in Childhood (ISAAC) [20] at the ages of 1, 2, 3 and 4 years. Response rates for these questionnaires were $71 \%, 76 \%, 72 \%$ and $73 \%$, respectively [21].

\section{Covariates}

Information on maternal age, parity, ethnicity, socioeconomic status, history of asthma or atopy and pet keeping was obtained by questionnaires completed by the mother during pregnancy. We used parity as a proxy for siblings, the correlation between those variables was good (Cohen's kappa 0.87; $\mathrm{p}<0.001$ ). 


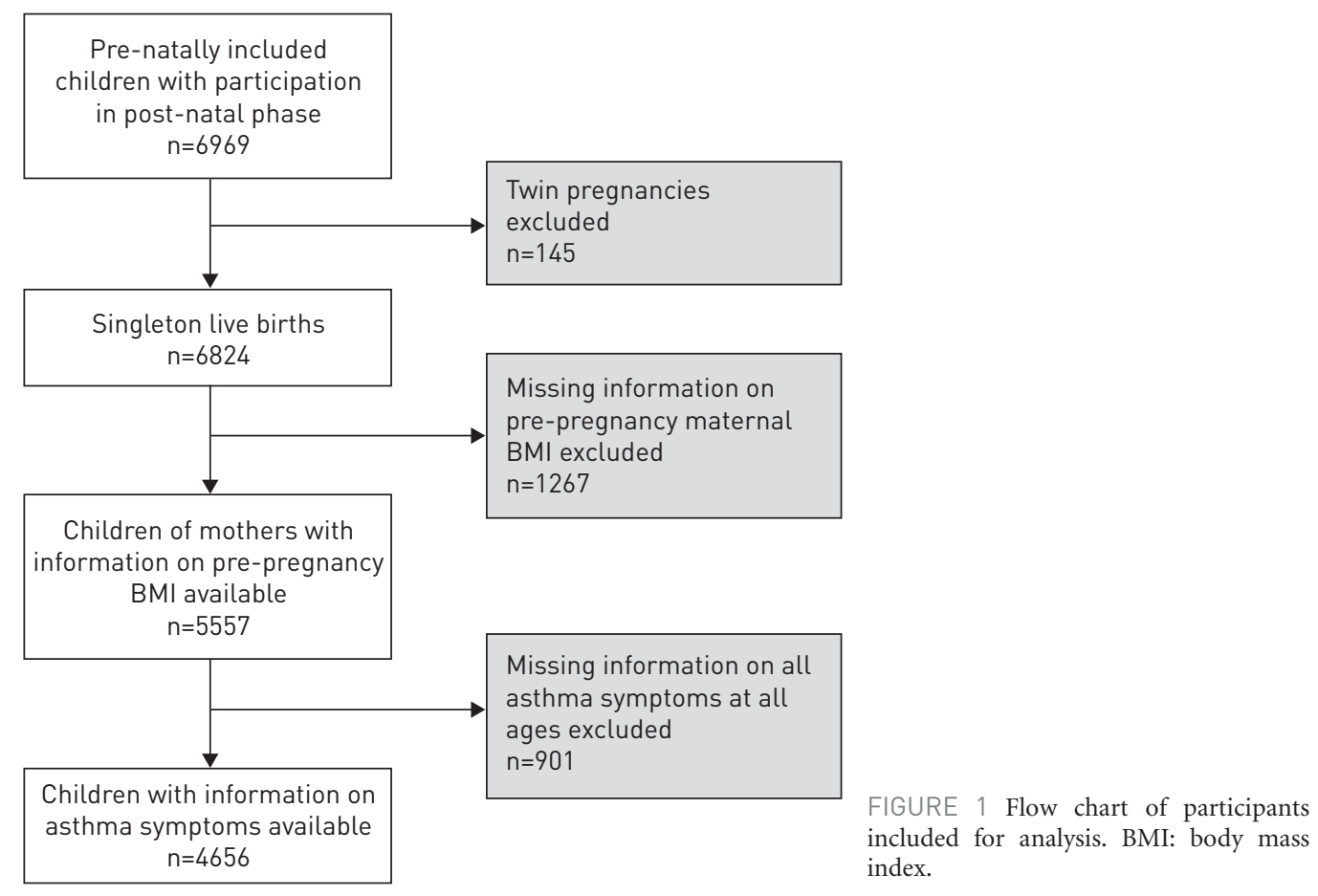

Maternal ethnicity was based on country of birth of her and her parents. Socioeconomic status was assessed using the highest maternal educational level. Information on maternal psychological distress was obtained by postal questionnaires at 20 weeks of gestation using the Brief Symptom Inventory [22]. Data on active maternal smoking was collected by postal questionnaires sent in the first, second and third trimester of pregnancy and combined into smoking (no or yes). Postal questionnaires at the ages of 6 and 12 months provided information about breastfeeding and daycare attendance, and at the ages of 1-4 years about lower respiratory tract infections (pertussis, bronchitis, bronchiolitis or pneumonia) and doctor-attended eczema $[18,21]$. Weight and gestational age at birth and sex of the children were obtained from midwife and hospital registries. The presence of gestational diabetes and hypertensive disorders was retrieved from birth records after delivery. Height and weight at the ages 1-4 years were measured at the child healthcare centre between 10 and 13, 23 and 29, 35 and 44 and 44 and 56 months of age, respectively [18].

\section{Statistical analysis}

We analysed the associations of maternal pre-pregnancy BMI and gestational weight gain with wheezing at the ages of 1-4 years using generalised estimating equation models (GEEs). With GEE analyses, repeatedly measured symptoms over time can be analysed, taking into account that these repeated measurements within the same subject are correlated. To prevent bias associated with missing data, missing values of the covariates and the outcome were multiple imputed based on the correlation of the missing variables with other characteristics. 10 imputed data sets were created and analysed separately, after which results were combined. All models were first performed unadjusted and subsequently adjusted for potential confounders. Selection of confounders was based on previous studies, if the effect estimates changed by $\geqslant 5 \%$, or if they were strongly related with the outcomes of interest. To assess whether the associations of maternal pre-pregnancy BMI and weight gain during pregnancy with wheezing could be explained by growth, infectious or atopic mechanisms, we additionally adjusted the analyses for child's growth, including height and weight, lower respiratory tract infections and eczema at the corresponding ages. Furthermore, we stratified the analysis for maternal history of asthma or atopy, to explore differences in associations of prepregnancy BMI and gestational weight gain with wheezing between children with and without a predisposition for asthma. The statistical analyses were performed using the SPSS version 17.0 (SPSS Inc., Chicago, IL, USA) and SAS 9.2 (SAS institute, Cary, NC, USA).

\section{Results}

\section{Subject characteristics}

Of the mothers, $2740(58.8 \%)$ had a normal pre-pregnancy BMI, 727 (15.6\%) were underweight, $844(18.1 \%)$ were overweight and $345(7.4 \%)$ were obese (table 1). Mean gestational weight gain was mean \pm SD $10.4 \pm 4.7 \mathrm{~kg}$. Children were born after median pregnancy duration of 40.1 weeks (5-95\% range 37.1-42.1 weeks) 
TABLE 1 Characteristics of children and their mothers ${ }^{\#}$

Original data

$$
\begin{gathered}
30.8 \pm 4.8 \\
13.8(10.1-27.2)
\end{gathered}
$$

Gestational age at enrolment weeks

$$
\text { Parity }
$$

Nullipara

Multipara

Missing

Ethnicity

European

Non-European

Missing

Education

Primary or secondary

Higher

Missing

Stress during pregnancy (global severity index)

Smoking during pregnancy

No

Yes

Missing

History of asthma or atopy

No

Yes

Missing

Pet keeping

No

Yes

Missing

Gestational hypertensive disorders

No

Yes

Missing

Diabetes gravidarum

No

Yes

Missing

Pre-pregnancy BMI $\mathrm{kg} \cdot \mathrm{m}^{-2}$

Pre-pregnancy BMI

Underweight $\left(<20 \mathrm{~kg} \cdot \mathrm{m}^{-2}\right)$

Normal weight $\left(20-24.9 \mathrm{~kg} \cdot \mathrm{m}^{-2}\right)$

Overweight $\left(25-29.9 \mathrm{~kg} \cdot \mathrm{m}^{-2}\right)$

Obese ( $\left.\geqslant 30 \mathrm{~kg} \cdot \mathrm{m}^{-2}\right)$

Gestational weight gain kg

\section{Children's characteristics}

\section{Female sex}

Gestational age at birth weeks

Birthweight $g$

Breastfeeding

No

Yes

Missing

Daycare attendance 1st year

No

Yes

Missing

$$
\begin{gathered}
2721(58.5) \\
1933(41.5) \\
2(0.0) \\
3105(67.1) \\
1519(32.9) \\
32(0.7) \\
2161(47.3) \\
2409(52.7) \\
86(1.8)
\end{gathered}
$$

$0.13(0.00-1.25)$

$$
\begin{gathered}
3824(86.4) \\
603(13.6) \\
229(4.9)
\end{gathered}
$$

2567 (61.7)

1593 (38.3)

$496(10.7)$

$2884(66.5)$

$1456(33.5)$

316 (6.8)

$4271(94.0)$

271 (6.0)

$114(2.4)$

4500 (99.3)

$33(0.7)$

$123(2.6)$

$23.4 \pm 4.1$

727 (15.6)

$2740(58.8)$

844 (18.1)

$10.4 \pm 4.7$

$2326(50.0)$

$40.1(36.0-42.3)$

$3457 \pm 546$

339 (7.5)

4152 (92.5)

165 (3.5)

$1693(44.4)$

$2120(55.6)$

$843(18.1)$
345 (7.4)
Data after multiple imputation

$30.8 \pm 4.8$

$13.8(10.1-27.2)$

2722 (58.5)

1934 (41.5)

3119 (67.0)

1537 (33.0)

2228 (47.9)

2428 (52.1)

$0.16(0.00-1.17)$

4019 (86.3)

637 (13.7)

2935 (63.0)

1721 (37.0)

3101 (66.6)

1555 (33.4)

4370 (93.9)

286 (6.1)

4611 (99.0)

45 (0.9)

$23.4 \pm 4.1$

727 (15.6)

2740 (58.8)

844 (18.1)

345 (7.4)

$10.4 \pm 4.7$

$2326(50.0)$

40.1 (36.0-42.3)

$3457 \pm 546$

$352(7.6)$

4304 (92.4)

$2192(47.1)$

$2464(52.9)$

Values are presented as mean \pm SD, $n(\%)$ or median (95\% range). BMI: body mass index. ${ }^{\#}: \mathrm{n}=4656$; ${ }^{\circ}$ : missing information for stress during pregnancy was $14 \%$. 
with a mean \pm SD birthweight of $3457 \pm 546$ g. Wheezing prevalences declined from $29.3 \%$ in the first year to $13.7 \%$ in the fourth year (table 2). We observed that per category increase of pre-pregnancy BMI, mean gestational weight gain was lower (mean gestational weight gain $10.9 \mathrm{~kg}$ and $7.5 \mathrm{~kg}$ for underweight and obese females, respectively) (table S1).

\section{Pre-pregnancy BMI and wheezing}

We observed an association of maternal pre-pregnancy BMI with the risks of preschool wheezing at the age of 4 years $(\mathrm{p}<0.01)$. Other significant associations of maternal pre-pregnancy BMI or categories with wheezing at other ages were not observed (fig. 2). Additional stratified analysis on maternal history of asthma or atopy showed that pre-pregnancy obesity was only associated with overall risks of preschool wheezing among mothers with a history of asthma or atopy (OR 1.47, 95\% CI 1.12-1.95). The stratified analysis also showed that among mothers with a history of asthma or atopy, pre-pregnancy underweight tended to be associated with increased risk of preschool wheezing (OR 1.17, 95\% CI 0.97- 1.42) (table 3). Per year analysis showed that the associations with preschool wheezing in obese mothers were seen from the age of 2 years onwards (table S2). No association was observed among children from mothers without a history of asthma or atopy. The size of the effect estimates did not change after adjustment for child height and weight, lower respiratory infections or eczema.

\section{Gestational weight gain}

Weight gain during pregnancy was associated with a slightly increased risk of wheezing at the age of 1 year (OR 1.13, 95\% CI 1.05-1.21), per standard deviation increase of gestational weight gain, and an overall increased risk from 1-4 years (overall OR 1.09, 95\% CI 1.04-1.14), per standard deviation increase of weight gain (table 4). These effects were independent of pre-pregnancy BMI. After stratification for prepregnancy BMI, we observed that the effect of gestational weight gain on overall risks of wheezing was the strongest among pre-pregnant normal weight and overweight females (OR 1.08 (95\% CI 1.01-1.15) and OR 1.18 (95\% CI 1.06-1.31), respectively, per standard deviation increase of weight gain). Stratification for maternal history of asthma or atopy showed that the effect estimates for the association between gestational weight gain and preschool wheezing were similar among children from mothers with and without a history of asthma or atopy. Also, the test for interaction between gestational weight gain and maternal history of asthma or atopy was nonsignificant $(\mathrm{p}=0.29)$ (table 5). Additional adjustment for infant height and weight, lower respiratory tract infections and eczema at the corresponding ages did not alter our results (table 5 and table S3).

\section{Discussion}

Our results showed that maternal pre-pregnancy obesity was associated with an increased risk of wheezing in the child, mainly if mothers had a history of asthma or atopy. Gestational weight gain was associated with increased risks of preschool wheezing, independent of pre-pregnancy BMI. This association was strongest for wheezing at age 1 year and was not different between mothers with and without a history of asthma or atopy. The effect of maternal pre-pregnancy BMI and gestational weight gain on preschool wheezing could not be explained by a child's growth, infectious or atopic mechanisms.

Our study confirms previous studies reporting positive associations of maternal pre-pregnancy obesity with preschool wheezing in age groups varying from the neonatal period until adolescence [1-6]. A study of 33192 children in Norway reported an association between maternal BMI and wheezing in children up to the age of 18 months [5]. This association was also present in two US studies, where higher maternal BMI was associated with higher risks of recurrent wheezing and asthma diagnosis at age 3 years $[2,6]$. In the Netherlands, in a study of 3963 children, maternal BMI was associated with risk of asthma at age 8 years, only in children predisposed to asthma [1]. Large studies in North Europe showed that maternal weight also increased risks of asthma diagnosis among adolescents [4], but only among those without a parental history of asthma [3].

Previous studies that assessed the effect of maternal pre-pregnancy weight on childhood asthma did not take maternal gestational weight gain into account, except for one recent published study [23]. This study showed that both increased pre-pregnancy maternal weight and gestational weight gain, when mutually adjusted, were independently associated with offspring wheeze and asthma at 7 years. Our results are consistent with the findings of this study.

Pre-pregnancy BMI and gestational weight gain are both associated with an increased risk of gestational hypertensive disorders [24]. These pregnancy complications may explain the associations of pre-pregnancy BMI and gestational weight gain with childhood wheezing. However, adding these variables to the models did not materially change the effect estimates, suggesting that they do not explain the observed associations. 
TABLE 2 Prevalences of age-dependent child characteristics ${ }^{\#}$

\begin{tabular}{|c|c|c|c|c|}
\hline & \multicolumn{4}{|c|}{ Age } \\
\hline & 1 year & 2 years & 3 years & 4 years \\
\hline \multicolumn{5}{|l|}{ Wheezing } \\
\hline Yes & 1366 (29.3) & $946(20.6)$ & 643 (13.8) & $636(13.7)$ \\
\hline No & $3290(70.7)$ & 3710 (81.4) & 4013 (86.2) & 4020 (86.3) \\
\hline \multicolumn{5}{|l|}{ Eczema } \\
\hline Yes & 1015 (21.8) & 673 (14.5) & $449(9.6)$ & $392(8.4)$ \\
\hline No & 3641 (78.2) & 3983 (85.5) & $4207(90.4)$ & $4264(91.6)$ \\
\hline \multicolumn{5}{|l|}{ LRTI } \\
\hline Yes & 870 (18.7) & $321(6.9)$ & 435 (9.3) & $318(6.8)$ \\
\hline No & 3786 (81.3) & 4335 (93.1) & 4221 (90.7) & 4338 (93.2) \\
\hline Height $\mathrm{cm}$ & $74.4 \pm 2.7$ & $88.3 \pm 3.4$ & $97.4 \pm 3.8$ & $103.3 \pm 4.1$ \\
\hline Weight kg & $9.67 \pm 1.07$ & $12.95 \pm 1.50$ & $15.28 \pm 1.85$ & $17.00 \pm 2.16$ \\
\hline
\end{tabular}

Data are presented as $n(\%)$ or mean \pm SD. LRTI: lower respiratory tract infection. ${ }^{*}: n=4656$.

We hypothesised that the associations of pre-pregnancy BMI and gestational weight gain with wheezing in offspring could be explained by a child's growth, infectious and atopic mechanisms. Previous studies were not always able to adjust for a child's own weight in the analysis $[2,4,5]$. In the studies that did adjust for the weight of the child, the effect estimates of the associations of maternal BMI with asthma symptoms were only slightly attenuated, and remained significant $[1,3,6]$. Part of the association of maternal weight before and during pregnancy with childhood asthma might, however, still be explained by increased levels of adiposity-related inflammatory factors or total body fat. Although BMI is thought to be a valid proxy for fat mass in children [25], adjusting for height and weight of young children might not be sufficient and further research should focus on more direct measurements of body composition.

Childhood wheezing is a complex phenotype, which might be caused partly by both infectious [26] and atopic mechanisms. Neither infectious diseases nor eczema explained the associations of pre-pregnancy BMI and gestational weight gain with preschool wheezing. Familial predisposition did slightly modify the effects.

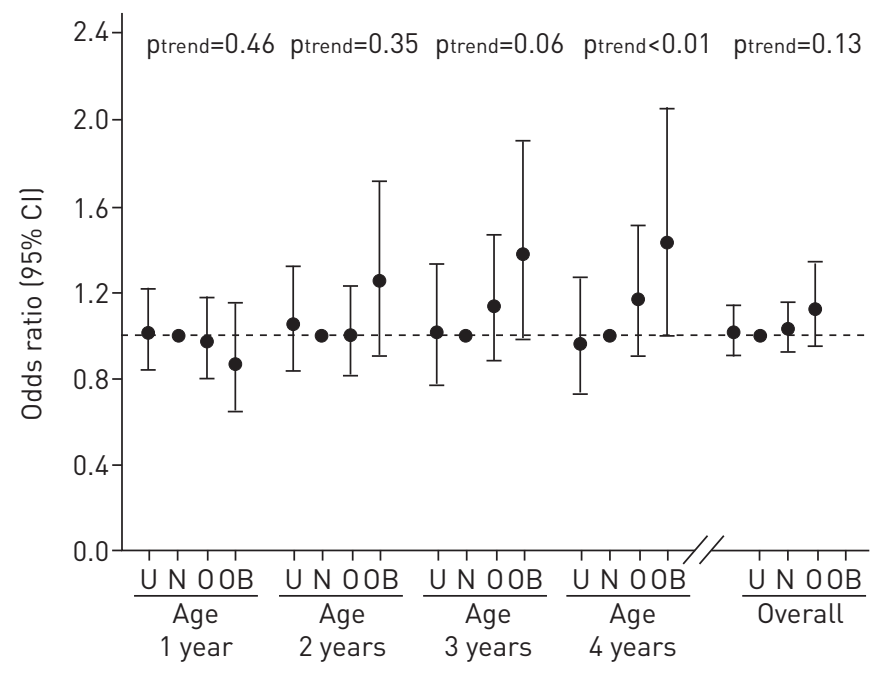

FIGURE 2 Associations of pre-pregnancy body mass index (BMI) (where U: underweight; N: normal weight; O: overweight; and OB: obese) with risks of wheezing $(n=4656)$. Data reflect the associations of different pre-pregnancy BMI groups with risks of wheezing, compared with the normal pre-pregnancy BMI weight group $\left(20-24.9 \mathrm{~kg} \cdot \mathrm{m}^{-2}\right) \mathrm{using}$ generalised estimating equation models. Tests for trend were based on generalised estimating equation models with prepregnancy $\mathrm{BMI} \pm \mathrm{SD}$ as a continuous variable. Models were adjusted for maternal age, parity, ethnicity, education level, distress during pregnancy, smoking during pregnancy, pet keeping, gestational hypertensive disorders, diabetes gravidarum, gestational age at enrolment, gestational age at measurement, gestational weight gain, and child's sex, gestational age at birth, birthweight, breastfeeding and daycare attendance. $p_{\text {trend }}$ : p-value for trend. 


\section{TABLE 3 Pre-pregnancy body mass index (BMI) and wheezing, stratified for maternal history of asthma or atopy\#}

Subjects $n$

Overall OR $(95 \% \mathrm{Cl})$

\begin{tabular}{|c|c|c|c|c|c|}
\hline & & & & & \\
\hline & & Model 1 & $\begin{array}{c}\text { Model } 2 \\
\text { (model 1+growth') }\end{array}$ & $\begin{array}{c}\text { Model } 3 \\
\text { (model 1+LRTI) }\end{array}$ & $\begin{array}{c}\text { Model } 4 \\
\text { (model } 1+\text { eczema) }\end{array}$ \\
\hline \multicolumn{6}{|c|}{ No maternal history of asthma or atopy } \\
\hline Normal weight & 1744 & Reference & Reference & Reference & Reference \\
\hline Overweight & 521 & $1.10(0.95-1.27)$ & $1.09(0.95-1.26)$ & $1.08(0.93-1.27)$ & $1.11(0.96-1.28)$ \\
\hline Obese & 217 & $0.94(0.74-1.19)$ & $0.93(0.73-1.19)$ & $0.89(0.69-1.15)$ & $0.93(0.74-1.19)$ \\
\hline Underweight & 275 & $1.17(0.97-1.42)$ & $1.19(0.98-1.43)$ & $1.23(1.01-1.50)^{*}$ & $1.17(0.97-1.42)$ \\
\hline Normal weight & 996 & Reference & Reference & Reference & Reference \\
\hline Overweight & 323 & $0.97(0.81-1.16)$ & $0.95(0.80-1.14)$ & $1.00(0.83-1.21)$ & $0.97(0.81-1.16)$ \\
\hline Obese & 128 & $1.47(1.12-1.95)^{* *}$ & $1.41(1.06-1.87)^{*}$ & $1.42(1.04-1.93)^{*}$ & $1.47(1.11-1.95)^{* *}$ \\
\hline Pre-pregnancy $\mathrm{BMI}^{+}$ & & $1.07(1.00-1.15)$ & $1.06(0.98-1.13)$ & $1.06(0.99-1.15)$ & $1.07(1.00-1.15)$ \\
\hline
\end{tabular}

Models were adjusted for maternal age, parity, ethnicity, education level, distress during pregnancy, smoking during pregnancy, pet keeping, gestational hypertensive disorders, diabetes gravidarum, gestational age at enrolment, gestational age at measurement, gestational weight gain, and child's sex, gestational age at birth, birthweight, breastfeeding and daycare attendance. LRTI: lower respiratory tract infections. ${ }^{\#}$ : $\mathrm{n}=4656$; ": growth was defined as child's height and weight at the ages of 1-4 years. Data reflect the associations of different pre-pregnancy BMI groups with wheezing of the children, compared with the normal pre-pregnancy BMI group, $20-24.9 \mathrm{~kg} \cdot \mathrm{m}^{-2}$, using generalised estimating equation models. ${ }^{*}: \mathrm{p}<0.05{ }^{* *}: \mathrm{p}<0.01$. Overall $\mathrm{p}_{\text {interaction }}$ pre-pregnancy $\mathrm{BMI} \times$ maternal history of asthma or atopy: 0.15 (for underweight $\mathrm{p}=0.08$, for overweight $p=0.42$, for obesity $p<0.011$.

It has been speculated that maternal overweight increases the risk of a child's nonatopic asthma only [27]. In contrast, some studies suggested that the effect of maternal obesity on childhood asthma symptoms was highest in children with a predisposition of asthma [1], but results seem inconsistent [3]. The role of infectious, atopic and familial predisposition remains inconclusive and need to be studied further in detail.

We showed that maternal history of atopy or asthma significantly modified the association between maternal pre-pregnancy BMI and preschool wheezing, but not between gestational weight gain and wheezing. Higher gestational weight gain was most strongly associated with preschool wheezing at age 1 year. The effects from pre-pregnancy BMI on childhood wheezing were only seen from the age of 2 years onwards, with a nonsignificant tendency towards an opposite effect for wheezing at age 1 year. Also, interaction between pre-pregnancy BMI and gestational weight gain was not significant $(p=0.64)$. These findings suggest that these associations of maternal pre-pregnancy BMI and gestational weight gain on childhood wheezing operate through different underlying mechanisms.

A potential underlying mechanism could be the role of leptin, a hormone produced by adipocytes and by the placenta. Higher BMI has been associated with higher leptin levels in pregnant females [13]. Leptin receptors are present in the fetal lung and may contribute to lung development in utero [28]. Also, leptin stimulates the production of pro-inflammatory cytokines, which might affect the development of the fetal immune system [13]. Further studies focused on the role of leptin in the associations of maternal prepregnancy BMI and gestational weight gain with preschool wheezing are needed.

Some methodological strengths and limitations need to be considered. This study was embedded in a population-based prospective cohort study with a large number of subjects being studied from early fetal life onwards with detailed prospectively and repeatedly measured information on maternal weight and wheezing, and a large number of potential confounders and mediating factors available. The response rate at baseline for participation in the Generation R Study cohort was $61 \%$. This nonresponse would lead to biased effect estimates if the associations differed between those included and not included in the analyses. However, this seems unlikely because biased estimates in large cohort studies mainly arise from loss to follow-up rather than from nonresponse at baseline [29]. Furthermore, we imputed missing data to prevent possible selection bias due to loss to follow-up, which minimised biased effect estimates due to selective response on measurements. Information on maternal pre-pregnancy weight was self-reported. Self-reported weight tends to be underestimated. However, in our study, self-reported pre-pregnancy weight was strongly correlated with weight measured at enrolment $(\mathrm{r}=0.95)$. Although systematic misclassification could not be fully excluded, we 
TABLE 4 Associations of gestational weight gain and risks of wheezing, in the total population and per maternal pre-pregnancy body mass index (BMI) category ${ }^{\#}$

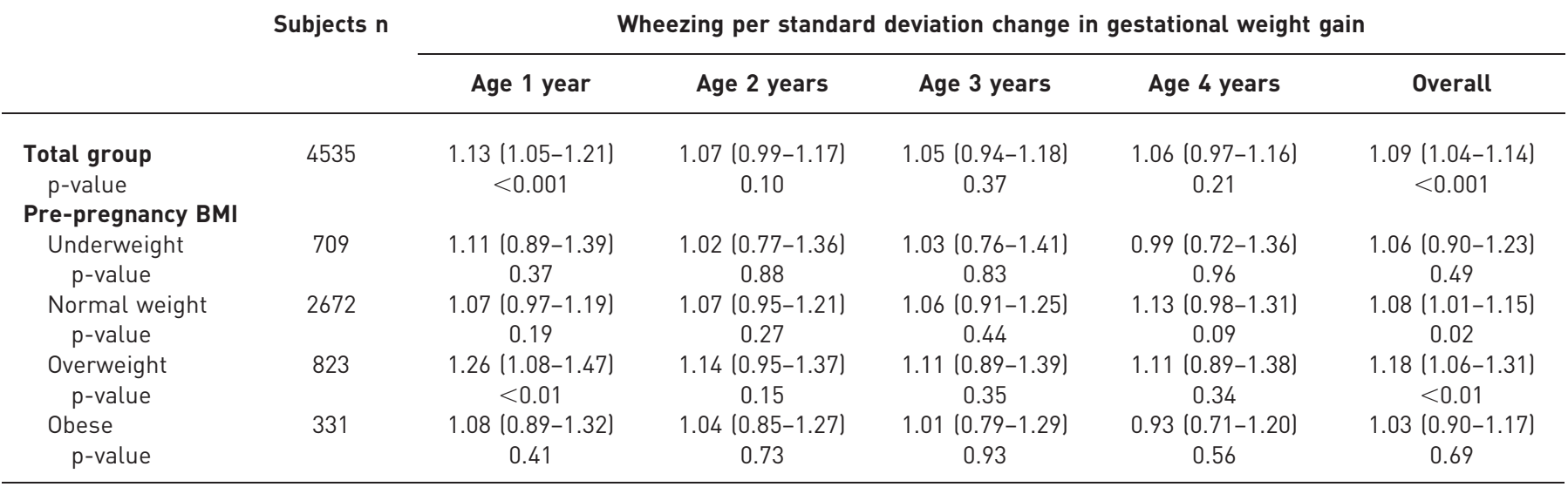

Data are presented as odds ratio ( $95 \%$ confidence interval), unless otherwise stated. Data were based on generalised estimating equation models with gestational weight gain per standard deviation as a continuous variable and reflect the association with wheezing per standard deviation increase of gestational weight gain. Models were adjusted for maternal age, parity, ethnicity, education level, distress during pregnancy, history of asthma or atopy, smoking during pregnancy, pet keeping, gestational hypertensive disorders, diabetes gravidarum, gestational age at enrolment, gestational age at measurement, and child's sex, gestational age at birth, birthweight, breastfeeding and daycare attendance. Analyses in the total group were adjusted for maternal pre-pregnancy BMI. Overall $p_{\text {interaction }}$ pre-pregnancy BMI $\times$ gestational weight gain $=0.64$. ${ }^{\#}: \mathrm{n}=4535$.

TABLE 5 Associations of gestational weight gain and risks of wheezing, stratified for maternal history of asthma or atopy

Subjects $n$

Overall OR $(95 \%$ CI)

do not expect that this explains the findings in our study. Furthermore, we also observed associations of maternal pre-pregnancy BMI with childhood wheezing when BMI was used as a continuous variable.

Wheezing prevalences were based on maternal reports using ISAAC questionnaires, the method that is widely accepted in epidemiological studies and reliably reflects the incidence of wheezing in young children [30]. It should be considered that maternal awareness and interpretation could lead to misclassification of the outcome if normal-weight mothers reported differently than overweight or obese mothers. Although we adjusted for several potential confounders, residual confounding due to unmeasured or insufficiently

\begin{tabular}{|c|c|c|c|c|}
\hline & Model 1 & $\begin{array}{c}\text { Model } 2 \\
\text { (model 1+growth") }\end{array}$ & $\begin{array}{c}\text { Model } 3 \\
\text { (model 1+LRTI) }\end{array}$ & $\begin{array}{c}\text { Model } 4 \\
\text { (model 1+eczema) }\end{array}$ \\
\hline \multicolumn{5}{|l|}{2864} \\
\hline \multirow{4}{*}{1671} & $1.10(1.04-1.16)$ & $1.10(1.04-1.16)$ & $1.09(1.03-1.16)$ & $1.10(1.03-1.16)$ \\
\hline & $<0.01$ & $<0.01$ & $<0.01$ & $<0.01$ \\
\hline & $1.09(1.01-1.17)$ & $1.08(1.01-1.16)$ & $1.07(1.00-1.16)$ & $1.09(1.01-1.17)$ \\
\hline & 0.02 & 0.03 & 0.06 & 0.02 \\
\hline
\end{tabular}

No maternal history of
asthma or atopy
Weight gain per standard
deviation increase
p-value
Maternal history of asthm
or atopy
Weight gain per standard
deviation
p-value

Values were based on generalised estimating equation models with gestational weight gain per standard deviation as a continuous variable and reflect the association with wheezing per standard deviation increase of gestational weight gain. ${ }^{\#}: \mathrm{n}=4535$; " growth defined as child's height and weight at the age at the ages of 1 to 4 years. Models were adjusted for maternal age, parity, ethnicity, education level, distress during pregnancy, smoking during pregnancy, parity, pet keeping, gestational hypertensive disorders, diabetes gravidarum, gestational age at enrolment, prepregnancy BMI, and child's sex, gestational age at birth, birthweight, breastfeeding and daycare attendance. Overall $p_{\text {interaction }}$ (gestational weight gain $\times$ maternal history of asthma or atopy $=0.29$. 
measured sociodemographic and lifestyle-related determinants might still be an issue, as in any observational study.

Our findings suggest that children of mothers with pre-pregnancy obesity and a history of asthma or atopy, and children of mothers with a higher gestational weight gain had higher risks of preschool wheezing. This association could not be explained by child's growth, infectious or atopic mechanisms. Given the high prevalence and considerable impact of childhood asthma on morbidity and healthcare costs, a causal pathway between maternal weight and preschool wheezing would be of great importance for public health. Therefore, further research is needed to identify the underlying mechanisms and long-term consequences. Also, new preventive strategies for pre-pregnant obese females should be developed aiming at reducing various adverse health outcomes in their children, including the burden of obstructive lung disease.

\section{Acknowledgements}

The Generation R Study is conducted by the Erasmus Medical Centre in close collaboration with the School of Law and the Faculty of Social Sciences at the Erasmus University, Rotterdam, the Municipal Health Service, Rotterdam area, and the Stichting Trombosedienst and Artsenlaboratorium Rijnmond (Star-MDC), Rotterdam, the Netherlands. We gratefully acknowledge the contribution of general practitioners, hospitals, midwives and pharamacies in Rotterdam.

\section{References}

1 Scholtens S, Wijga AH, Brunekreef B, et al. Maternal overweight before pregnancy and asthma in offspring followed for 8 years. Int J Obes (Lond) 2010; 34: 606-613.

2 Reichman NE, Nepomnyaschy L. Maternal pre-pregnancy obesity and diagnosis of asthma in offspring at age 3 years. Matern Child Health J 2008; 12: 725-733.

3 Patel SP, Rodriguez A, Little MP, et al. Associations between pre-pregnancy obesity and asthma symptoms in adolescents. J Epidemiol Community Health 2012; 66: 809-814.

4 Lowe A, Braback L, Ekeus C, et al. Maternal obesity during pregnancy as a risk for early-life asthma. J Allergy Clin Immunol 2011; 128: 1107-1109.

5 Haberg SE, Stigum H, London SJ, et al. Maternal obesity in pregnancy and respiratory health in early childhood. Paediatr Perinat Epidemiol 2009; 23: 352-362.

6 Kumar R, Story RE, Pongracic JA, et al. Maternal pre-pregnancy obesity and recurrent wheezing in early childhood. Pediatr Allergy Immunol Pulmonol 2010; 23: 183-190.

7 McDonald SD, Han Z, Mulla S, et al. Overweight and obesity in mothers and risk of preterm birth and low birth weight infants: systematic review and meta-analyses. BMJ 2010; 341: c3428.

8 Ay L, Kruithof CJ, Bakker R, et al. Maternal anthropometrics are associated with fetal size in different periods of pregnancy and at birth. The Generation R Study. BJOG 2009; 116: 953-963.

9 Caudri D, Wijga A, Gehring U, et al. Respiratory symptoms in the first 7 years of life and birth weight at term: the PIAMA Birth Cohort. Am J Respir Crit Care Med 2007; 175: 1078-1085.

10 Hancox RJ, Poulton R, Greene JM, et al. Associations between birth weight, early childhood weight gain and adult lung function. Thorax 2009; 64: 228-232.

11 Canoy D, Pekkanen J, Elliott P, et al. Early growth and adult respiratory function in men and women followed from the fetal period to adulthood. Thorax 2007; 62: 396-402.

12 Sonnenschein-van der Voort AM, Jaddoe VW, Raat H, et al. Fetal and infant growth and asthma symptoms in preschool children: the Generation R Study. Am J Respir Crit Care Med 2012; 185: 731-737.

13 Hendler I, Blackwell SC, Mehta SH, et al. The levels of leptin, adiponectin, and resistin in normal weight, overweight, and obese pregnant women with and without preeclampsia. Am J Obstet Gynecol 2005; 193: 979-983.

14 Guler N, Kirerleri E, Ones U, et al. Leptin: does it have any role in childhood asthma? J Allergy Clin Immunol 2004; 114: 254-259.

15 Stuebe AM, McElrath TF, Thadhani R, et al. Second trimester insulin resistance, early pregnancy body mass index and gestational weight gain. Matern Child Health J 2010; 14: 254-260.

16 Beuther DA, Weiss ST, Sutherland ER. Obesity and asthma. Am J Respir Crit Care Med 2006; 174: 112-119.

17 Jaddoe VW, van Duijn CM, Franco OH, et al. The Generation R Study: design and cohort update 2012. Eur J Epidemiol 2012; 27: 739-756.

18 Jaddoe VW, van Duijn CM, van der Heijden AJ, et al. The Generation R Study: design and cohort update 2010. Eur J Epidemiol 2010; 25: 823-841.

19 Gaillard R, Steegers EA, Hofman A, et al. Associations of maternal obesity with blood pressure and the risks of gestational hypertensive disorders. The Generation R Study. J Hypertens 2011; 29: 937-944.

20 Asher MI, Keil U, Anderson HR, et al. International Study of Asthma and Allergies in Childhood (ISAAC): rationale and methods. Eur Respir J 1995; 8: 483-491.

21 Sonnenschein-van der Voort AM, Jaddoe VV, van der Valk RJ, et al. Duration and exclusiveness of breastfeeding and childhood asthma-related symptoms. Eur Respir J 2012; 39: 81-89.

22 De Beurs E, Zitman FG. De Brief Symptom Inventory (BSI): De betrouwbaarheid en validiteit van een handzaam alternatief voor de SCL-90. [The Brief Symptom Inventory (BSI): The reliability and validity of a brief alternative of the SCL-90.] Maandblad Geestelijke Volksgezondheid 2006; 61: 120-141.

23 Harpsoe MC, Basit S, Bager P, et al. Maternal obesity, gestational weight gain, and risk of asthma and atopic disease in offspring: a study within the Danish National Birth Cohort. J Allergy Clin Immunol 2013; 131: 1033-1040.

24 Gaillard R, Durmuş B, Hofman A, et al. Risk factors and outcomes of maternal obesity and excessive weight gain during pregnancy. Obesity 2013 [In press DOI: 10.1002/oby.20088].

25 Pietrobelli A, Faith MS, Allison DB, et al. Body mass index as a measure of adiposity among children and adolescents: a validation study. J Pediatr 1998; 132: 204-210.

26 Wright AL. Epidemiology of asthma and recurrent wheeze in childhood. Clin Rev Allergy Immunol 2002; 22: 33-44.

27 Weiss ST. Obesity: insight into the origins of asthma. Nat Immunol 2005; 6: 537-539. 
Gnanalingham MG, Mostyn A, Gardner DS, et al. Developmental regulation of the lung in preparation for life after birth: hormonal and nutritional manipulation of local glucocorticoid action and uncoupling protein-2. J Endocrinol 2006; 188: 375-386.

29 Nohr EA, Frydenberg M, Henriksen TB, et al. Does low participation in cohort studies induce bias? Epidemiology 2006; 17: 413-418.

30 Jenkins MA, Clarke JR, Carlin JB, et al. Validation of questionnaire and bronchial hyperresponsiveness against respiratory physician assessment in the diagnosis of asthma. Int J Epidemiol 1996; 25: 609-616. 